\title{
Universal non-equilibrium phenomena at submicrometric surfaces and interfaces
}

\author{
R. Cuerno ${ }^{1}$, M. Castro ${ }^{2}$, J. Muñoz-García, R. Gago ${ }^{3}$, and L. Vázquez ${ }^{4}$ \\ 1 Departamento de Matemáticas and Grupo Interdisciplinar de Sistemas Complejos (GISC), \\ Universidad Carlos III de Madrid, 28911 Leganés, Spain \\ 2 Escuela Técnica Superior de Ingeniería and GISC, Universidad Pontificia de Comillas, \\ 28015 Madrid, Spain \\ 3 Centro de Micro-Análisis de Materiales, Universidad Autónoma de Madrid, 28049 Madrid, Spain \\ 4 Instituto de Ciencia de Materiales de Madrid (CSIC), 28049 Madrid, Spain
}

\begin{abstract}
The recent widespread interest in processes occurring at micro and nanometric scales has increased the physical relevance of the surfaces and interfaces constituting system boundaries, both at and far from equilibrium. In the latter case, universal properties occur, such as scale invariance (surface kinetic roughening), surface pattern formation or domain coarsening. However, descriptions of these systems feature limited predictive power when based merely on universality principles. We review examples from Materials Science at nano and submicrometric scales, that underlie the importance of describing growing surfaces by means of (phenomenological) constitutive laws, in order to correctly describe the rich behaviors experimentally found across many different systems. Additionally, this approach provides new generic models that are also of interest in the wider contexts of Pattern Formation and Non-Linear Science.
\end{abstract}

In memoriam C. Pérez García

\section{Introduction}

The progressive focus of Condensed Matter Physics towards smaller scales, micro or even nanometric, implies that surfaces and interfaces have to play an ever more important role for the systems under study. At equilibrium, this is merely the result of an increased surface to volume ratio, which, for systems with a finite number of particles, leads to a non negligible contribution from surface effects to the free energy [1]. In the case of far-from-equilibrium systems, such as for instance growing systems, the dynamical relevance of the surface is clear, since growth events often take place at the surface itself. These considerations are valid both for inorganic [2] and for biological systems [3], submicrometric scales conspicuously providing a common playground for both type of systems (see an example of a nanometric sized biological interface in figure 1, to which the theoretical tools to be described below are fully applicable). The study of surface and interface dynamics thus has an increased interest due to their basic aspects, as instances of spatially extended dynamical systems [4], and also for their wide scope applications in other contexts ranging from nanotechnology to biosystems. In Statistical Physics, indeed, the subject of surface dynamics has become increasingly important in the last few years as a paradigmatic field to look for a variety of genuine non-equilibrium phenomena, whose interplay can be often studied under an unified perspective. Thus, one finds generic scale invariance and multiscaling, morphological instabilities, pattern coarsening and many other phenomena $[5,6]$. A remarkable consequence of the universality of these phenomena is their ubiquity in 


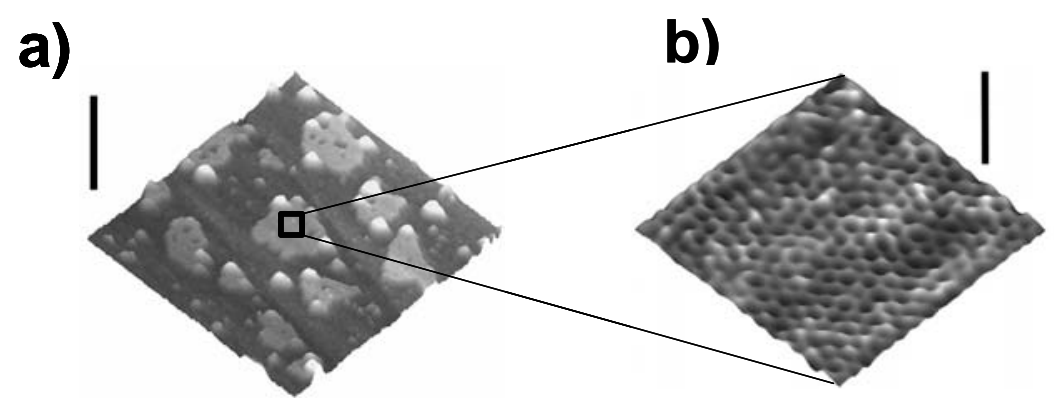

Fig. 1. $2 \times 2 \mu \mathrm{m}^{2}$ AFM image of surface morphology of Sticholysin II (StnII) crystals formed on lipid monolayers of 1,2-dioleoyl-sn-glycero-3-phosphatidylcholine (DOPC) deposited on a highly oriented pyrolytic graphite substrate. (b) $82 \times 82 \mathrm{~nm}^{2}$ AFM image, filtered by low-pass FFT, of the surface of the crystal marked in (a). The vertical bars correspond to $100 \mathrm{~nm}$ (a) and $8 \mathrm{~nm}$ (b).

experimental situations, which in principle enables the use of common tools to describe physical processes taking place at spatial length scales differing in up to seven orders of magnitude. For instance, one can think of the nano-structures produced during ion-beam bombardment of (amorphous) solid targets and relate them to pattern formation in aeolian sand dunes $[7,8]$ (see more on this example in section 3.2.2 below). Other contexts, in which concepts and techniques derived from non-equilibrium interface dynamics are relevant, include typical problems like the growth of thin films by chemical or physical vapor deposition or Molecular Beam Epitaxy [9], the properties of biological membranes and cellular aggregates [10], as well as viscous fluids at macroscopic and microscopic scales [11]. Nevertheless, when attempts have been made at comparison between specific experiments and predictions from models built mostly upon universality principles (thus, largely independent of growth system specifics), the latter have frequently featured unsatisfactory predictive power (see e.g. [12] for a recent overview).

The themes of this contribution are, first, the success of continuum approaches in the description of many global properties of surfaces and interfaces at submicrometric scales [13]. Second, the importance of describing the new relevant physical mechanisms arising by means of appropriate (phenomenological) constitutive laws [14] that can in principle accommodate more specific (as opposed to universal) system properties. Many times these laws can indeed be established thanks to the high accuracy of current experimental techniques to measure surface properties [15], underlying the importance of combined experimental and theoretical approaches. Third, the discovery through this route of new phenomena and models that feature universal properties and can thus be expected to apply to domains beyond submicron Materials Science, such as those providing more traditional applications of Pattern Formation and Nonlinear Science at large: e.g. fluids, granular systems, etc. The present contribution is organized as follows. In section 2 we consider three paradigmatic classes of non-equilibrium surface phenomena in which the notion of universality is relevant. We will choose a specific thin film growth/erosion process to illustrate each of them. Section 3 will be devoted to continuum approaches to describe these systems, wherein we will review successes and limitations of "top-down" approaches working from universality principles and more "down-top" procedures that start from constitutive laws. In order to illustrate the latter, we will revisit the thin film production techniques considered previously. We will be led to new universal models and phenomena that arise simultaneously in several of them and elsewhere, that will be described in section 4 , after which we offer some conclusions and an outlook in section 5 .

\section{Universal (surface) properties}

Recent Materials Science and Condensed Matter Physics have witnessed the discovery of interesting physical properties of the surfaces and interfaces arising e.g. in many techniques for thin film production, either by growth or by erosion or etching of a solid aggretgate/substrate. 
From a technological point of view, these properties may affect device performance, e.g. a high interface roughness leading to poor electric or mechanical contact. Thus, efficient production techniques are seeked in which good understanding of the conditions under which optimal morphological properties can be achieved. From a more basic perspective, these systems uniquely provide instances of non-equilibrium systems in which interesting global phenomena occur that, to a large extent, (i) are material independent, (ii) display the same qualitative features across preparation/fabrication techniques and (iii) show a strong dependence on dimensionality/kinetic constraints. All these features, together with the high precision data that are provided by current surface characterization techniques (scanning probe microscopies such as AFM and STM, synchrotron radiation, surface spectroscopies, etc.) [15] make them ideal case studies to which the analytical and conceptual tools of Statistical Mechanics and Non-Linear Science seem particularly well suited.

\subsection{Surface disorder (kinetic roughening)}

As a first example, we consider thin film production by Chemical Vapor Deposition (CVD), which is routinely employed e.g. in the fabrication of (opto)electronic devices and, more recently, for microfluidic cells [16]. Within a simplified view [17], in a standard CVD experiment a mixture of reactive species in gas phase is introduced into a furnace, onto whose walls a (macroscopically sized) stagnant diffusive layer forms. Reactive species diffuse across this layer and eventually may incorporate onto the surface of a growing aggregate through a series of chemical reactions. Examples of $\mathrm{SiO}_{2}$ thin films grown as in [18] are shown in figure 2. Panel (a) shows the aggregate surface after $50 \mathrm{~min}$. deposition time, while panel (b) is a snapshot taken after 2 days deposition. The horizontal and vertical scales have been resized (anisotropically in the horizontal and vertical directions) in such a way that both images have the same statistical properties, thus providing an explicit example of surface scale invariance. The associated disorder in the height values across the images, together with the non-equilibrium origin of these morphological features, have led to the expression "kinetic roughening" to denote this spatiotemporal behavior of the surface height. One way to quantify it is through the power spectral density $P S D(k, t)=\left\langle h_{k}(t) h_{-k}(t)\right\rangle$, where $h_{k}(t)$ is the Fourier transform of the local deviation of the height field $h(x, t)$ around its mean value. This is plotted in panel (c) of figure 2 for the surfaces of panels (a) and (b). The power-law behavior of $P S D(k)$ with wave-vector $k$ seen in figure 2(c) is completely analogous to that of e.g. the local coarse-grained order parameter (spontaneous magnetization) of an Ising model, in equilibrium at the critical temperature; moreover, considering the time evolution of the curves in figure 2(c), we can think of the critical dynamics of the magnetization [19]; indeed, the universality classes associated with the values of the critical exponents arising in kinetic roughening $[5,12]$ generalize to far-from-equilibrium situations the more familiar classes of equilibrium critical dynamics [20]. In this sense, kinetic roughening becomes an instance of the wider phenomenon of "generic scale invariance" [21].
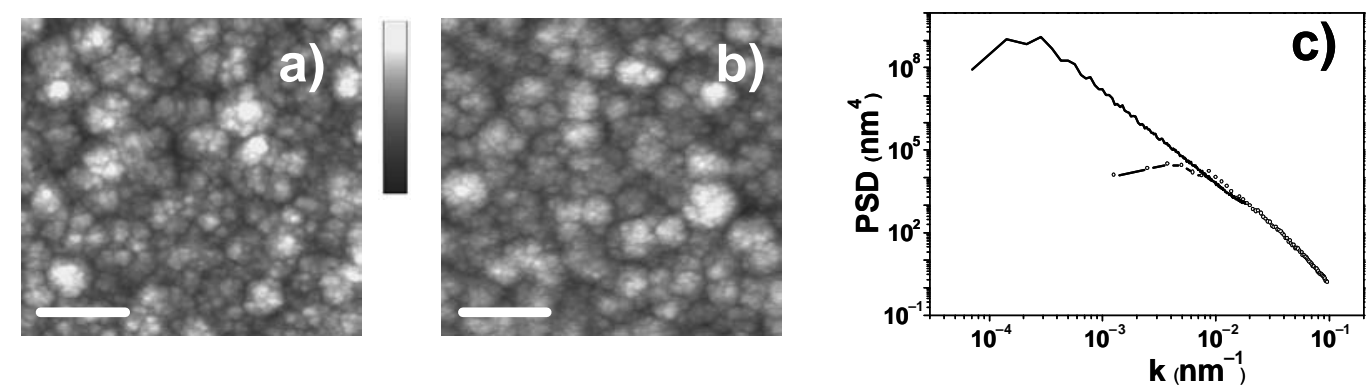

Fig. 2. AFM top views of $\mathrm{SiO}_{2}$ films grown by thermal CVD onto silicon substrates for $50 \mathrm{~min}$. (a) and 2 days (b). The horizontal bars correspond to $195 \mathrm{~nm}$ (a) and $4.1 \mu \mathrm{m}$ (b), the vertical greyscale ranging from 0 up to $22 \mathrm{~nm}$ (a) or $190 \mathrm{~nm}$ (b). Panel (c) shows logarithmic plots of the PSD functions vs. wave-vector $k$, computed for the original data of panels (a) and (b) bottom to top, respectively. 

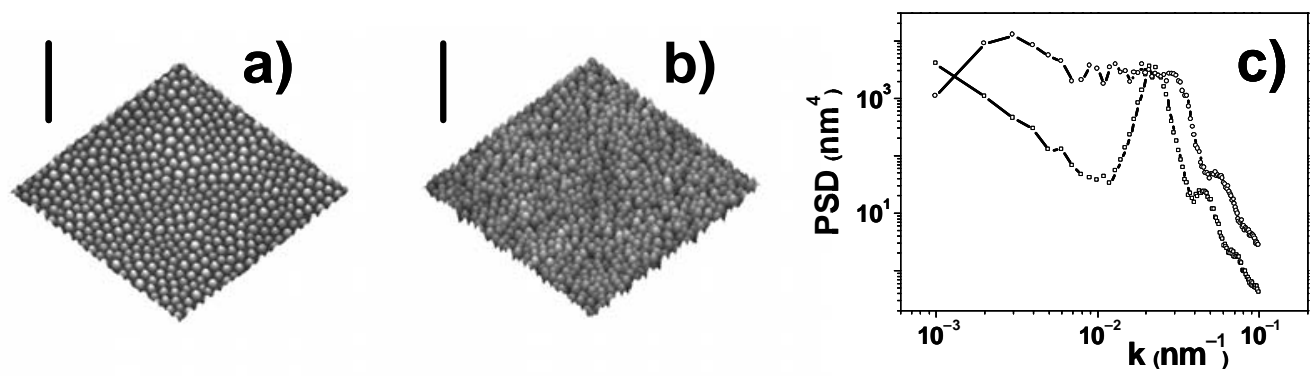

Fig. 3. $1 \times 1 \mu \mathrm{m}^{2}$ AFM images showing the nanodot pattern morphology induced by IBS with argon ions onto different types of targets: (a) silicon for $1 \mathrm{keV}$ ions; (b) GaSb for $0.5 \mathrm{keV}$ ions. The vertical bars correspond to $100 \mathrm{~nm}$ (a) and $200 \mathrm{~nm}$ (b). Panel (c) shows the PSD functions computed for panels (a) and (b) bottom to top, respectively.

\subsection{Surface pattern formation}

While kinetic roughening is associated with the lack of typical time and length scales in the evolution of the surface morphology, it is of course possible (to some extent, almost unavoidably $[22])$ to encounter experimental situations in which surface features are characterized by well defined time and length scales, the morphology displaying a well defined pattern as a result of some morphological instability [23]. This is the case, for instance, in the nanostructuring of many materials by ion-beam sputtering (IBS). In these experiments, a target is irradiated by a collimated beam of energetic ions (typical energies being in the keV range) that impinge onto the former under a well defined angle of incidence. Although routinely employed since long for many diverse applications within Materials Science (material implantation, sample preparation, etc.) the capabilities of this technique for efficient nanopatterning have been recognized only recently [24]. Thus, it induces self-organized regular ripple or dot nanopatterns over large areas (up to $1 \mathrm{~cm}^{2}$ ) onto metal [24], semiconductor, and insulator surfaces [25] after a few minutes of irradiation. In figure 3 we show dot patterns produced in IBS experiments similar to those in [25], onto silicon and GaSb targets. Interestingly, the main features of this pattern formation process seem to be largely independent of the type of ions and targets employed (as long as the latter amorphizes under irradiation), which encourages use of the standard tools of Pattern Formation $[6,26]$ to address description and modelling of these systems. Thus, topographical analysis of samples by various types of microscopies (AFM, TEM, SEM) focusing on the dynamics of observables such as the PSD or the height autocorrelation [27] allow to assess the (hexagonal, square) symmetry of the in-plane ordering of the nanostructures, as well as the time evolution of its characteristics (wavelength coarsening at intermediate times) and some of its substrate-specific features (lenticular vs cone or sinusoidal shaped cross section). For instance, figure 3(c) shows the PSD functions computed for the experimental images shown in panels (a), (b) of the same figure. The main peak (whose existence obviously breaks scale invariance) corresponds to the dot size, while secondary peaks indicate the short-range lateral ordering in the structure. Necessarily, theoretical approaches to this phenomenon relate strongly with paradigmatic universal models within the field of Pattern Formation, such as the KuramotoSivashinsky or the Benney equations, and their generalizations [28].

\subsection{Domain coarsening}

Our third example of universality concerns the dynamics of a morphological feature (such as e.g. a pattern wavelength, or the average surface slope) that grows as a power law of time and, thus, evolves in the absence of a typical time scale. This type of far-from-equilibrium behavior is, again, strongly reminiscent of more familiar phenomena that relate with equilibrium models, such as phase ordering or domain growth, e.g. the coarsening of spins domains in an Ising model after a quench from a temperature above critical, down to $T<T_{c}[29,30]$. In 

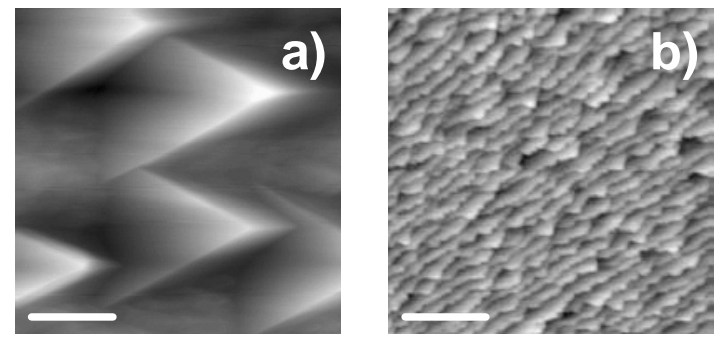

Fig. 4. GaAs(110) vicinal surfaces misoriented towards (111). (a) $5 \times 5 \mu \mathrm{m}^{2}$ image of mounds. (b) $3 \times 3 \mu \mathrm{m}^{2}$ image of rough steps between terraces. The horizontal bars correspond to $1.25 \mu \mathrm{m}$ (a) and $750 \mathrm{~nm}$ (b). Both images, courtesy of Dr. P. Tejedor, ICMM-CSIC.

our context, interesting examples of this type of behavior can be found within the context of crystalline thin film production by Molecular Beam Epitaxy (MBE), both in the case of growth of a surface with a high-symmetry crystalline orientation (singular surfaces) and when growing surfaces that are close (vicinal) to a high symmetry one. As examples of the latter, we show in figure 4(a) [31] an AFM top view of mounds that have evolved after a coarsening process in the growth of GaAs(110) vicinal surfaces misoriented towards (111), while panel (b) in the same figure shows a similar growth process [32] in which single monolayer steps can be appreciated, together with their rough 1D profiles. In growth onto singular surface orientations, for coverages (i.e., time) that are large enough, islands that have nucleated onto a high symmetry surface in the submonolayer regime start to coalesce and, due to specific microscopic mechanisms known to lead to morphological instabilities, such as the EhrlichSchwoebel (ES) barrier to adatom diffusion at down steps $[2,9,33]$, large surface protrusions (mounds) build up, whose average slope increases as a power law of time, $m(t)=\langle|\nabla h(\boldsymbol{x}, t)|\rangle \sim$ $t^{p}$. figure 4(a) shows an intermediate morphology, only that the initial surface was vicinal, rather than singular. Actually, the earlier stage of this surface shown in figure 4(b) allows us to appreciate in more detail the extent to which the surface morphology is due to the evolution of steps bordering terraces composing the vicinal surface. Thus, adatoms on terraces are subject to various physical mechanisms, mostly terrace and line diffusion, and ES barriers to step or kink down crossings, the step shape being the result of the interplay between adatom fluxes originating from each of the adjacent terraces. Again, the various diffusive phenomena give rise generically to morphological instabilities $[2,9,33]$, many times inducing a selected wavelength $\ell$ on the step line profile that the reader may appreciate in figure 4(b). Under many experimental conditions [see e.g. [34] for vicinal $\mathrm{Si}(001)$ substrates], this wavelength coarsens as a power law of time $\ell \sim t^{n}$. Thus, the two coarsening exponents $p, n$ characterize the growth laws of relevant morphological features in MBE experiments. Although the extent to which universality can be claimed in this context is not well understood yet, there are indeed measurements of these exponents under diverse conditions (see a recent overview in [9]), as well as proposed theoretical descriptions for which connection to classical domain ordering (Ostwald ripening) is a relevant guiding tool [35].

\section{Continuum approaches}

Although the atomistic physical mechanisms involved in the experiments mentioned above take place at scales around $1 \mathrm{~nm}$ or smaller and times around $1 \mathrm{ps}$, the morphological properties we have been describing occur on length scales that are above $10 \mathrm{~nm}$ and timescales around $1 \mathrm{~s}$. Due to this length scale separation, continuum descriptions may provide global, accurate, as well as computationally efficient, frameworks to describe these non-equilibrium phenomena, with a similar spirit to the Navier-Stokes equations for fluid motion. Moreover, recent developments allow to incorporate noise into mesoscopic descriptions in a consistent way [36], so that the domain of applicability of such improved descriptions is currently being extended down to even smaller scales. Continuing with an example from Fluid Dynamics, a recent example is 
the noisy generalization in [37] of the lubrication approximation [11]. At present such type of phenomenological generalizations of continuum theories are finding a surprisingly wide range of applicability.

In general, seeking the most compact continuum description, many theoretical efforts are directed towards the formulation of a single closed evolution equation for the interface height that can be either deterministic or stochastic.

\section{1 “Universal” approaches: Limitations}

Within the assumption of universality in any of the above contexts, where this notion seems relevant, one can hope to "derive" valid continuum descriptions (i.e. interface equations) based only on symmetry considerations, combined e.g. with scaling arguments of the appropriate type. However, many times this procedure applies at best at the asymptotic state so that one has to be careful if the goal is to produce an equation that can account for preasymptotic features which under many conditions happen to be the ones to which experimental runs are feasible. A paradigmatic example is provided by kinetic roughening, wherein the Kardar-Parisi-Zhang (KPZ) equation (equivalent to the stochastic Burgers equation) is in principle expected to describe the interface fluctuations whenever growth takes place in the absence of conservation laws for the height field. In spite of this expectation, paradoxically very few experiments have been reported in which KPZ exponents have been consistently measured (see one example in [18], and overviews in [12,22]). Moreover, the problem with this type of "derivations" is that they do not enable a detailed connection with phenomenological parameters describing specific experimental systems. In the context of kinetic roughening, these facts led to invoking additional effects on a coarse-grained level, such as exotic noise statistics, non-local effects, etc. [5], in order to account for the difference between the observed and the predicted scaling behaviors of rough surfaces. However, a wide range of scaling exponents ensued, there being no theoretical argument that could identify the correct exponents for a specific growth experiment. Moreover, no apparent cause for the experimental scarcity of KPZ scaling could be provided. An example is provided by erosion by IBS, in which symmetry considerations suggest a simple KPZ description, which would miss the physical morphological instability that leads to the nanopattern formation in these systems. This suggests, as is also the case e.g. for CVD, that the difficulty in observing the asymptotic KPZ behavior is the long crossover induced by the existing morphological instabilities. However, these are preasymptotic features, beyond description through mere symmetry/scaling arguments. Therefore, a different approach is required, which allows to trace back each contribution in the interface equation to specific relaxation mechanisms and which allows to select the most physically relevant terms that are present in a specific type of experiments, within the implied coarse-grained description.

\subsection{Phenomenological approaches}

We will thus consider alternative continuum approaches that are more phenomenological in nature, some of which have been reviewed elsewhere [13,14]. Typically, these start out by establishing approximate constitutive equations (possibly with allowance for noise contributions) that govern dynamics in these non-equilibrium systems. To this end, there being new physical effects acting at these reduced scales, close relation to actual experiments is required, which benefits from the high quality of data that current surface analysis techniques can provide. This allows to make contact between physical (experimental) and model parameters. Once this is done, the ensuing description has typically the form of a (stochastic) moving boundary (MB) problem. Already at this stage, relevant information can be obtained, although typically MB problems are hard to tackle both analytically and numerically. In order to proceed further, two (complementary) approaches can be taken. On the analytical side, building upon the scale separation mentioned above that typically occurs in these systems, it is many times possible to perform some sort of weakly non-linear expansion ending up in a single closed interface equation. Such a simplified model possibly has some sort of generality or universality, in the sense 
of the previous paragraph but, remarkably, provides a more complete description of the system dynamics by reflecting the existing morphological instabilities, crossovers (preasymptotic scaling behavior) and, possibly, the relevant (albeit perhaps practically unobservable) asymptotic behavior. From the numerical side, one can resort to descriptions that, being equivalent to the MB problem of reference, are amenable to more efficient and accurate numerical simulations. We can mention diffuse interface (equivalently, phase-field) [38] and kinetic Monte Carlo models [39]. In what follows we will mostly review some recent examples of the first analytical approach, combined with occasional mention of the second type of more computationally oriented studies.

\subsubsection{Kinetic roughening: Chemical vapor deposition}

As a first example we revisit thin film growth by CVD. The relevant MB description is provided by the following set of coupled equations [40]

$$
\begin{aligned}
& \partial_{t} c=\nabla \cdot(D \nabla c-\mathbf{q}), \\
& (D \nabla c-\mathbf{q}) \cdot \mathbf{n}=\left.k_{D}\left(c-c_{e q}^{0}-\Gamma \kappa+\chi\right)\right|_{h}, \\
& \mathbf{v} \cdot \mathbf{n}=\Omega[D \nabla c-\mathbf{q}] \cdot \mathbf{n}, \\
& \lim _{z \rightarrow \infty} c(x, z ; t)=c_{a},
\end{aligned}
$$

of which (1) describes diffusion of vapor species (with concentration $c$ ) in an infinite stagnant layer, (4) providing the driving boundary condition at the layer edge. The efficiency of the diffusive flux contribution to aggregate growth is accounted for by the mixed boundary condition (2) at the aggregate surface $h$, with $\Gamma$ being the surface tension, $\kappa$ the mean surface curvature, $k_{D}$ a kinetic coefficient related with the sticking probability for aggregating units, and $\mathbf{n}$ giving the local surface normal direction. Finally, the aggregate velocity $\mathbf{v}$ is provided in (3) by that part of the diffusive flux that effectively attaches to the solid surface where, for the sake of simplicity, here we have neglected surface diffusion effects, see [40]. Model (1)-(4) incorporates fluctuations (noise) $\mathbf{q}$ and $\chi$ to the diffusive current and deposition events, respectively, thus generalizing the otherwise classic description of CVD [41]. Moreover, as shown in [40], this very same $\mathrm{MB}$ model describes a class of aggregate growth experiments by electrochemical deposition (ECD). Carrying on the program described at the beginning of section 3.2 , a small slope approximation of (1)-(4) leads to different interface equations as a function of the kinetic coefficient $k_{D}$; thus, for $k_{D} \rightarrow 0$ (namely, low sticking probability), the interface equation is local, and reads

$$
\partial_{t} h=v+\frac{v}{2}(\nabla h)^{2}-|\nu| \nabla^{2} h-B \nabla^{2}\left(\nabla^{2} h\right)+\eta,
$$

which is nothing else than the celebrated Kuramoto-Sivashinsky equation [42], a paradigm of spatiotemporal chaos within Nonlinear Science; however, a large sticking probability $\left(k_{D} \rightarrow \infty\right)$ leads, rather, to an equation that is nonlocal in space, namely,

$$
\partial_{t} h_{k}=\left[v|k|\left(1-a_{3} k^{2}\right)-B k^{4}\right] h_{k}+\frac{v}{2} \text { Fourier Transf. }\left[\left(\partial_{x} h\right)^{2}\right]+\eta_{k} .
$$

Both in equations (5) and (6), the $\eta$ terms are the corresponding noise contributions, that are additive to this order of approximation. The behaviors predicted by equations (5) and (6) have many qualitative similarities: in both cases, there is a short time, short length scale morphological instability induced by the diffusive contribution to the growth process by which peaks on the aggregate surface "shadow" and grow at the expense of troughs. This unstable interface dynamics is dominated by a typical wavelength that corresponds to the surface Fourier mode growing at the fastest rate. At long enough times, surface slopes are large enough so that non-linear effects can no longer be neglected, and these are such that the morphology disorders in height values so that the long distance behavior can be described in an effective way by stochastic equations that feature kinetic roughening (e.g. the KPZ equation for the case of (5) [44]). Thus we see in particular how surface order (pattern formation) or disorder (kinetic 

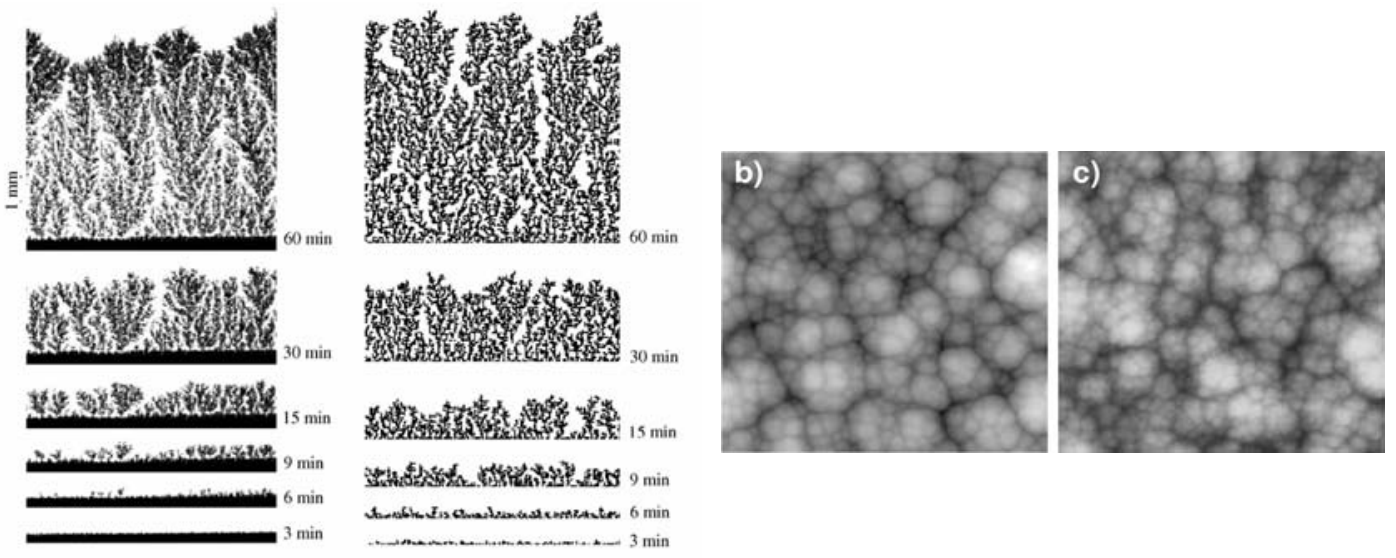

Fig. 5. (a) (Left) Electrochemical deposits of Ag from [47], by kind permission of the authors. (Right) MBDLA simulations after [46]. (b) Numerical simulation of equation (6) for a two-dimensional substrate. (c) $1 \times 1 \mathrm{\mu m}^{2}$ AFM snapshot of hydrogenated amorphous carbon films grown by electron cyclotron resonance.

roughening) can coexist, each of these phenomena applying at different scales for a given system. Moreover, kinetic roughening is seen at the asymptotic state which indeed occurs only after an extremely long crossover time for e.g. the KS system.

A more detailed study [43] reveals nevertheless some quantitative differences between equations (5) and (6), including the nature of the morphological instability as well as the universality class that characterizes the corresponding asymptotic state. Specifically, in the case of equation (6), the latter features a large value of the Hurst or roughness exponent. This is a signal of large average slopes across the surface, requiring an improved study that is not restricted by the small slope approximation. Whereas a detailed phase-field study is under way [45], we can appreciate some of the features of the dynamics predicted by the full MB model in the simulations shown on figure 5. Specifically, panel (a) in the figure illustrates the aggregate dynamics as described by a kinetic Monte Carlo type model (termed Multiparticle Biased Diffusion Limited Aggregation, MBDLA) studied in [46], and believed to provide a "discretized" formulation of (1)-(4). As mentioned above, this MB description also applies to growth by electrochemical deposition (ECD), panel (a) in figure 5 supporting this claim by additionally showing experimental results of Ag deposition [47] in parallel with the MBDLA data. In a sense, this validity across deposition techniques is a type of universality for the continuum description provided by the above MB problem. In the context of CVD proper, the model also provides an accurate description, as illustrated by the comparison in figure 5 between growth experiments of hydrogenated amorphous carbon films grown by electron cyclotron resonance CVD [panel (c)] and numerical simulations [43] of equation (6) for a two-dimensional substrate [panel (b)]. Quantitative comparison with experiments of the type shown in figure 5 is mostly beyond reach for equations derived from mere universality arguments [48], which typically would rule out some of the (required) asymptotically irrelevant terms from equations (5) and (6) to begin with.

Naturally, and although there are other thin film growth techniques that are even conceptually close to CVD and ECD, the MB problem seen above cannot be expected to account for surface dynamics in arbitrary surface growth problems. A wide class of processes that remain to be described is that in which the dominant transport mechanism of reacting species to the aggregate is ballistic, rather than diffusive as was the case in (1)-(4). A conspicuous example is provided by growth by sputtering, in which a material that is eroded from a solid target is eventually deposited onto a different substrate, see refs. in e.g. [49]. Actually, the type of scale invariance found frequently in these systems [50], known as anomalous scaling in this context, is not similar to equilibrium dynamic scaling [51], and remains to be understood from a fundamental point of view. 


\subsubsection{Pattern formation: Ion-beam sputtering}

As an example of the modelling of nanopattern formation by means of constitutive laws we take target erosion by ion-beam sputtering. For bombarding ions with energies around $1 \mathrm{keV}$ and amorphous or amorphizable targets, it was Sigmund [52] who clarified the physical origin of the morphological instability induced by irradiation as due to local surface minima being eroded with a larger rate than surface maxima. Later phenomenological approaches [53] incorporated this morphological mechanism, combined with stabilizing processes such as surface diffusion, into an effective interface equation that is an anisotropic (reflecting the loss of symmetry between substrate directions induced by the ion-beam) generalization of the KS equation. However, these generalizations [28] failed at reproducing the short range order properties of the nanopatterns observed, as well as the coarsening (precisely in the sense of section 2.3) of the main pattern wavelength during intermediate times [24,25]. Recall the role of the KS equation as a paradigm of spatiotemporal chaos. Moreover, it is seen that higher-order corrections to the ensuing equations within the same approach lead to mathematically ill-posed interface equations [54]. Thus, additional physical mechanisms are needed in order to account for the experimental morphologies. To date, the procedure that seems to achieve a larger predictive power to this end $[7,8]$ couples the surface height field $h(\mathbf{r}, t)$ with an extra field $R(\mathbf{r}, t)$ that measures de density of mobile species at the surface into a set of rate equations

$$
\begin{aligned}
\partial_{t} h & =-\Gamma_{\mathrm{er}}+\Gamma_{\mathrm{ad}}, \\
\partial_{t} R & =(1-\phi) \Gamma_{\mathrm{er}}-\Gamma_{\mathrm{ad}}+D \nabla^{2} R,
\end{aligned}
$$

where $\Gamma_{\mathrm{er}}, \Gamma_{\mathrm{ad}}$ are rates of production of mobile surface species and of aggregation of adatoms to the solid surface, respectively, that depend on the local surface geometry (i.e. on $h$ and its derivatives) just as previous studies predict $[28,53]$, and also on the local density of mobile species $R$. In equation (8), $\phi$ is a parameter measuring the fraction of dislodged species that are irreversibly lost (sputtered), and $D$ is the surface diffusivity. Here we have neglected an advective term describing erosion by the so-called direct knock-on collisions between ions and mobile species [7], that are typically irrelevant under the experimental conditions we consider here. Note that, for $\phi=0$, the set of equations (7)-(8) conserves the total amount of material. This is actually the relevant physical situation in the context of pattern (ripple) formation on aeolian sand dunes, where so-called "hydrodynamic" models like the above have recently proved successful [55]. In our present context, the smallness of the typical erosion rate $\left(\simeq 1 \mathrm{~s}^{-1}\right)$ as compared with the adatom diffusion rate $\left(\simeq 1 \mathrm{ps}^{-1}\right)$ allows to eliminate the $R$ field adiabatically near threshold of the morphological instability and obtain perturbatively the following interface equation at normal incidence [7]

$$
\partial_{t} h=-\nu \nabla^{2} h-\mathcal{K} \nabla^{4} h+\lambda^{(1)}(\nabla h)^{2}+\lambda^{(2)} \nabla^{2}(\nabla h)^{2},
$$

that generalizes as follows under oblique ion incidence [8]

$$
\begin{aligned}
\partial_{t} h= & \gamma \partial_{x} h+\sum_{i=x, y}\left\{-\nu_{i} \partial_{i}^{2} h+\lambda_{i}^{(1)}\left(\partial_{i} h\right)^{2}+\Omega_{i} \partial_{i}^{2} \partial_{x} h+\xi_{i}\left(\partial_{x} h\right)\left(\partial_{i}^{2} h\right)\right\} \\
& +\sum_{i, j=x, y}\left\{-\mathcal{K}_{i j} \partial_{i}^{2} \partial_{j}^{2} h+\lambda_{i j}^{(2)} \partial_{i}^{2}\left(\partial_{j} h\right)^{2}\right\}
\end{aligned}
$$

In equations (9) and (10), all parameters depend on phenomenological constants (average ion energy and range, flux, angle of incidence, etc.). Note that, while the type of terms that appear in each case might be guessed by symmetry and scaling arguments, the signs of their coefficients cannot, so that the existence and properties of the physical morphological instability, and even the conditions under which (9) and (10) provide mathematically sound descriptions [54], are beyond reach for that type of arguments. Moreover, equations (9) and (10) are able to account for a wider range of experimental features than the KS and related equations that they generalize. Thus, figure 6(b) shows the stationary morphology of a Si target irradiated 

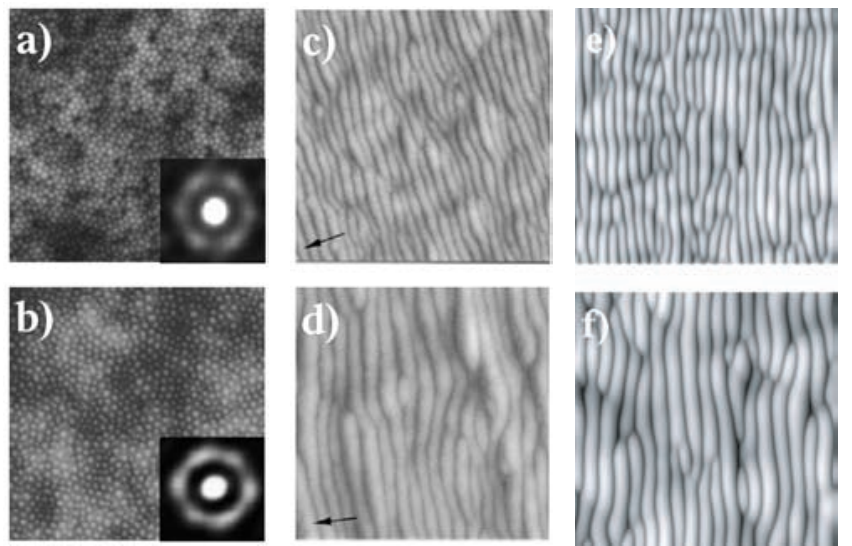

Fig. 6. (a) Stationary morphology of a Si target after irradiation under normal incidence as in [25](c) for $4 \mathrm{~h}$. Inset shows the corresponding height autocorrelation. (b) Stationary state as obtained by numerical integration of equation (9). (c), (d) $1 \times 1 \mu \mathrm{m}^{2}$ AFM views of fused silica targets irradiated under oblique incidence after 10 and 60 min. respectively. (e), (f) Early and late time morphologies, as predicted by numerical integration of equation (10). Panels (a), (b) are reproduced from [7], and (c), (d) are taken from [57], all by permission.

for $4 \mathrm{~h}$., which is favorably compared in panel (a) of the same figure with the result provided by numerical integration of equation (9). The main stages of evolution are similar to those described for the KS equation (5), with the difference that now there is coarsening of the main pattern wavelength at intermediate times (not shown in the figure, see also [56]) and the dot pattern shows short-range hexagonal ordering, as evidenced by the height autocorrelation shown in figures 6(a) and (b). The wavelength coarsening prior to the stationary state can be perhaps more easily visualized under oblique incidence conditions, when the pattern has a rippled shape. In figures 6(c) and (d) we show two AFM snapshots of the surface dynamics for fused silica undergoing bombardment at oblique incidence [57]. The black arrows represent the projection of the ion beam direction onto the target plane. The relatively ordered ripple structure clearly coarsens in time, as occurs in the case of the numerical integration of equation (10) shown on panels (e), (f) of the same figure. In general, a close study of the examples shown on figure 6 reveals the extent to which nanopatterning by IBS gives rise to the three types of universal morphological phenomena mentioned in section 2, within the evolution of a single system. Thus, a pattern formation process occurs at short times induced by a morphological instability, which introduces a typical length scale in the system, $\ell$. This scale evolves non-linearly during intermediate times in a coarsening process characterized at least by effective power laws $\ell \sim t^{n}$. Finally, the non-linear evolution of these structures gives rise to some degree of disordering, reminiscent of the KS chaos, in such a way that the height PSD shows power law behavior (kinetic roughening) for distances that are much larger than the ripple wavelength. We will come back soon to this remarkably rich behavior.

\subsubsection{Domain coarsening: Epitaxial growth of vicinal surfaces}

In order to illustrate domain coarsening in yet a different physical context, we consider dynamics of steps at vicinal surfaces growing by Molecular Beam Epitaxy. Following the seminal work of Burton, Cabrera and Frank [2,9], a MB problem can be formulated in which diffusion of adatoms takes place on terraces, step motion being due to the resulting diffusive fluxes at the step. In the absence of adatom evaporation on terraces and for a constant driving adatom flux $F$ on the terraces, one has the following MB problem [58]:

$$
\begin{aligned}
0 & =\nabla \cdot\left(D \nabla c_{j}\right)+F, \\
D \nabla c_{j} \cdot \mathbf{n} & =k_{D}^{(+)}\left[c_{j}-c_{j-1}^{(\text {eq })}\left(1+\Gamma \kappa_{j-1}\right)\right] \quad \text { at step } j-1,
\end{aligned}
$$




$$
\begin{aligned}
D \nabla c_{j} \cdot \mathbf{n} & =-k_{D}^{(-)}\left[c_{j}-c_{j}^{(\mathrm{eq})}\left(1+\Gamma \kappa_{j}\right)\right] \quad \text { at step } j, \\
\mathbf{v}_{j} \cdot \mathbf{n} & =\Omega\left(D \nabla c_{j+1}-D \nabla c_{j}\right) \cdot \mathbf{n},
\end{aligned}
$$

where $c_{j}$ is the adatom concentration on terrace $j$, which terminates at steps $j-1$ and $j$. For this reason, the analog of the mixed boundary condition (2) is provided by the two conditions (12) and (13). Otherwise, the problem (11)-(14) (and the interpretation of the physical constants appearing) is analogous to the MB problem seen in section 3.2.1, with a few important differences. First, now we have a problem of several coupled one-dimensional interfaces, namely, the monoatomic steps. Second, due to the Ehrlich-Schwoebel effect the kinetic coefficients $k_{D}^{( \pm)}$ for adatom attachment from the different sides of a step may now differ, which is the physical origin for the morphological (known as meandering) instability, first elucidated by Bales and Zangwill [59]. Within a quasistationary approximation in which concentration at terraces is taken to relax faster than any other process and assuming a weak ES effect, it has recently been shown [58] that the effective interface equation for an isolated step has the following form in non-dimensional units:

$$
\partial_{t} h=-\mu \nabla^{2} h-\mathcal{B} \nabla^{4} h+\lambda^{(2)} \nabla^{2}(\nabla h)^{2} .
$$

Equation (15), which has been termed a conserved Kuramoto-Sivashinsky equation, can be considered as e.g. the $\lambda^{(1)}=0$ case of equation (9), and has been derived in other physical contexts, such as ripple formation on aeolian sand dunes [55] (c), or amorphous thin film growth [60].

\section{New universal phenomena and models}

Work entering the phenomenology behind surface dynamics at submicron scales has allowed us to uncover an equation such as equaiton (9) that, in dimensionless units and for the simplest case of one-dimensional substrates [61], can be conveniently written as

$$
\partial_{t} h=-\partial_{x}^{2} h-\partial_{x}^{4} h+\left(\partial_{x} h\right)^{2}-r \partial_{x}^{2}\left(\partial_{x} h\right)^{2},
$$

depending on a single dimensionless parameter $r$ that can be seen to provide the (squared) ratio of a linear crossover length scale to a non-linear crossover length scale. Two-dimensional versions of equation (16) have appeared in the mesoscopic description of diverse systems, such as erosion by IBS $[7,61]$ or growth of amorphous thin films with inhomogeneities (in this case, within a noisy generalization) [62]. Interestingly, equation (16) allows us to systematically study within a single continuum model the interplay between pattern-formation (order) and fluctuations (disorder) via time-intermediate coarsening, namely, the interplay among the three classes of interface universal phenomena considered in section 2. Note that, as shown perhaps more clearly through the links between the (microscopic) IBS context with macroscopic pattern formation processes such as ripples on aeolian sand dunes, equation (16) might be relevant also to dynamics of macroscopic systems. equations (9), (16) interpolate between the KS (for $r=0$ ) and the conserved KS [simply take $\lambda^{(2)}=0$ in (9)] limits, as illustrated in figure 7 (a), where we show stationary morphologies from numerical simulations of equation (16) for several $r$ values. Note how the KS-type disordered cell morphologies for small $r$ yield to much larger ordered domains of cells for increasing $r$. For a fixed generic value of $r$, dynamics are thus intermediate between what each of the low and high $r$-limits would imply. Thus [61], the dominant wavelength of the cell morphology that appears at short times coarsens with time as dictated by the conserved KS behavior. This is because the associated nonlinearity minimizes a "chemical potential" $\mu(h)$ of the form $\mu=-\left(\partial_{x} h\right)^{2}$, as can be seen by writing its contribution to (16) as a continuity equation, $\partial_{t} h=-\partial_{x} j(x, t)$, with the current $j(x, t)=-\partial_{x} \mu$. Thus, once the linear sinusoidal pattern appears, the surface tries to have as few stationary ponits (where $\left.\partial_{x} h=0\right)$ as possible. This happens until slopes are large enough so that the non-conserved nonlinearity enters the dynamics non-trivially and induces a behavior that is reminiscent of the non-conserved KS equation. Thus, coarsening interrupts and the thus far ordered morphology 


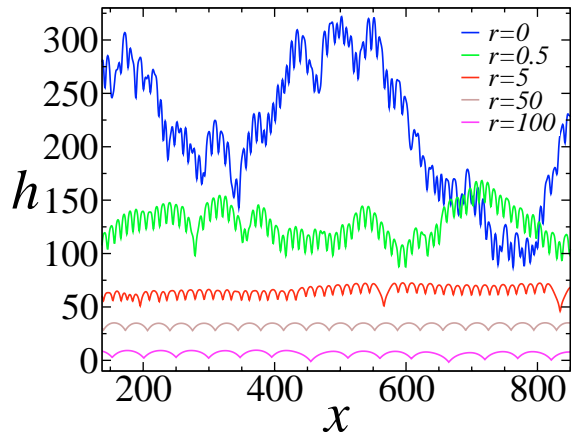

Fig. 7. Stationary morphologies from numerical simulations of equation (16), for values of $r$ as indicated.

eventually disorders in heights, to the extent that kinetic roughening properties appear in the fluctuation spectrum (PSD) of the surface for length scales that are much larger than the single cell size [44]. Remarkably, very large domains of ordered cells remain at intermediate scales, a behavior that provides a mild violation of Krug's conjecture [63] that "local height equations generally cannot describe the emergence and evolution of patterns of constant wavelength and amplitude". This statement obviously poses a potential limitation for the predictive power of (local) interfacial equations to describe experimentally observed morphologies, so that its elucidation is an important open issue in our context. Clues in this sense have been recently provided in [64], where a classification has been put forward for possible scenarios for one-dimensional coarsening, that include: (i) No coarsening (e.g. for the Ginzburg-Landau equation); (ii) spatiotemporal chaos (e.g. the KS equation, or the convective Cahn-Hilliard $(\mathrm{CH})[65]$ equation in some parameter range); (iii) perpetual coarsening (as in the conserved KS equation, or the convective $\mathrm{CH}$ equation for a different parameter range) and (iv) interrupted coarsening, namely, transient wavelength coarsening followed by unbounded amplitude growth, as in equations for step dynamics on vicinal surfaces without desorption in the strong ES effect limit [66] or in the case of $2 \mathrm{D}$ diffusion anisotropy in a similar context [67]. Note, the behavior of equation (16) does not fall within any of these possibilities, so that one can expect further work to be required in order to fully assess the descriptive capabilities of (local) interface equations.

\subsection{Alternative "universal" approaches}

The recent work just mentioned on the coarsening properties of interface equations has underlined the frequent appearance of this phenomenon in the dynamics of many submicrometric interfaces, naturally combined with pattern formation, and sometimes appearing as a preasymptotic feature prior to stationary surface scale invariance. Actually, building upon current (partial) knowledge on the type of non-linear terms that (and that do not) induce coarsening, one can supplement the standard symmetry and scaling arguments mentioned in section 3.1 in order to produce in a systematic way improved continuum descriptions that can account for preasymptotic features. An approach along these lines has been recently introduced in [68]. Briefly, if the physical growth process does not depend on a privileged height value, then the continuum description has to be invariant under uniform shifts of the form $h(\mathbf{r}, t) \rightarrow h(\mathbf{r}, t)+$ const. In principle, in the presence of morphological instabilities, the conservation law associated with this shift symmetry can lead to a large scale instability [69], which might prevent a unified description. Nevertheless, one can obtain in a systematic way the dominant and subdominant non-linear terms that are pertinent to a given system by casting its dynamics into a generalized relaxational form of the type

$$
\partial_{t} h=-\Gamma(\{h\}) \frac{\delta F(\{h\})}{\delta h},
$$

where a non-uniform mobility $\Gamma(\{h\})$ and a free-energy-type functional $F(\{h\})$ appear that are expanded in powers of height derivatives, and are strongly constrained by the shift symmetry [70]. In order to describe a given system, one can select which of the thus obtained non-linearities 


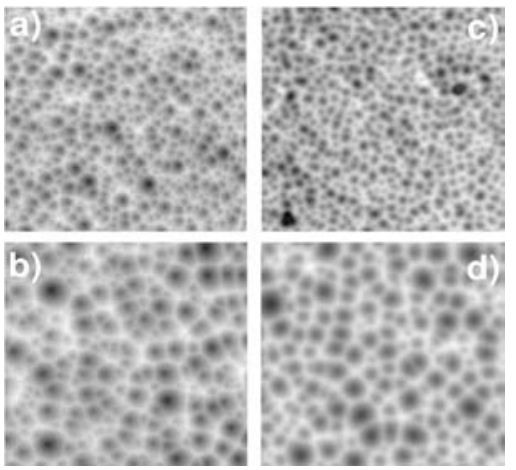

Fig. 8. (a)-(b) $50 \times 50 \mu \mathrm{m}^{2} \mathrm{AFM}$ top views of a $\mathrm{Si}(100)$ target undergoing erosion by immersion in an Ar plasma at early and late times, respectively. (c)-(d): Early and late time morphologies from simulations of equation (18), respectively.

are compatible with additional symmetries (e.g. reflection $\mathbf{r} \rightarrow-\mathbf{r}$ in the substrate plane, etc.), rank them in terms of relevance by means of scaling arguments and, finally, select subdominant terms according to the observed intermediate-time coarsening properties of the system. In reference [68] this program is not only seen to reproduce the relevant equations for many known systems, but also to lead to correct descriptions for new interface growth experiments. An example is provided by the agreement of data for erosion of a silicon target immersed in an Ar plasma, with the dynamics predicted by the following convective Cahn-Hilliard equation

$$
\partial_{t} h=-\nu \nabla^{2} h-K \nabla^{4} h+\lambda(\nabla h)^{2}+\lambda_{210}(\nabla h)^{2}\left(\nabla^{2} h\right),
$$

see figure 8 for a comparison of morphologies at the stationary state.

Analogous proposals exist in the recent literature [71] to produce continuum equations based on generalized multiscale arguments. Again, further work is needed in order to clarify the role of interface coarsening as a guiding principle in this context, as well as to assess the extent to which it provides a universal phenomenon, the universality classes it encompasses, the role of noise and dimensionality in the latter, etc.

\section{Conclusions and outlook}

As we have seen, phenomenological continuum approaches are proving rather successful in the description of universal and non-universal properties of the non-equilibrium dynamics of many surfaces and interfaces that appear in submicrometric systems. The new mechanisms that occur at these scales (increased relevance of surface vs bulk effects, new types of kinetic constraints, new system symmetries, etc.) require new modelling of the relevant constitutive laws. Somewhat surprisingly, continuum descriptions remain valid down to rather small scales while being able to probe, at the same time, the macroscopic time and length scales at which these systems show many interesting and non-trivial properties. The interface equations thus far obtained have allowed improved understanding of global phenomena such as e.g. kinetic surface roughening, and in particular their experimental significance. While these approaches are allowing a more quantitative description of interfacial phenomena at small scales, they are proving a rich source of non-equilibrium, non-linear models. Naturally, they still open interesting issues regarding e.g. the limits of applicability of continuum theory (supplemented by the addition of noise) to microscopic systems, the interplay of fluctuations (disorder) and pattern formation (order), and the somehow intermediate role and nature of coarsening processes. Answers to these questions may well be found to go much farther than the original contexts of Condensed Matter Physics where they are appearing, into the wider fields of Non-Linear and Complex Systems.

This contribution is dedicated to the lasting memory of the late Carlos Pérez García. We would like to thank $\mathrm{H}$. Mancini for his kind invitation to participate in the present issue.

We would also like to thank J. G. Buijnsters, M. García-Hernández, J. M. Mancheño, M. Nicoli, F. Ojeda, and P. Tejedor for various interactions. Our work has been partially supported by MECD 
(Spain), through Grants Nos. FIS2006-12253-C06-01, FIS2006-12253-C06-02, FIS2006-12253-C06-03, FIS2006-12253-C06-06, and by the Ramón y Cajal programme (R. G.).

\section{References}

1. T.L. Hill, Thermodynamics of Small Systems (Dover, New York, 1994)

2. A. Pimpinelli, J. Villain, Physics of Crystal Growth (Cambridge University Press, Cambridge, 1998)

3. S.A. Safran, Statistical Thermodynamics of Surfaces, Interfaces, and Membranes (Addison-Wesley, Reading, 1994)

4. J. García-Ojalvo, J.M. Sancho, Noise in Spatially Extended Systems (Springer-Verlag, New York, 1999)

5. A.-L. Barabási, H.E. Stanley, Fractal Concepts in Surface Growth (Cambridge University Press, Cambridge, 1995)

6. M.I. Rabinovich, A.B. Ezersky, P.D. Weidman, The Dynamics of Patterns (World Scientific, Singapore, 2000)

7. M. Castro, R. Cuerno, R. Gago, L. Vázquez, Phys. Rev. Lett. 94, 016102 (2005)

8. J. Muñoz-García, M. Castro, R. Cuerno, Phys. Rev. Lett. 96, 086101 (2006)

9. T. Michely, J. Krug, Islands, Mounds, and Atoms (Springer, Heidelberg, 2004)

10. Fluctuations and Scaling in Biology, edited by T. Vicsek (Oxford University Press, New York, 2001)

11. A. Oron, S. Davis, S.G. Bankoff, Rev. Mod. Phys. 69, 931 (1997); T.M. Squires, S.R. Quake, ibid. 77, 977 (2005)

12. R. Cuerno, L. Vázquez, in Advances in Condensed Matter and Statistical Physics, edited by E. Korutcheva, R. Cuerno (Nova Science, New York, 2004)

13. D.D. Vvedensky, J. Phys.: Condens. Matter 16, R1537 (2004)

14. J. Krug, in Collective Dynamics of Nonlinear and Disordered Systems, edited by G. Radons, P. Häussler, W. Just (Springer, Berlin, 2004)

15. Frontiers in Surface and Interface Science, edited by C.B. Duke and E.W. Plummer (Elsevier, Amsterdam, 2002)

16. P. Tabeling, Introduction to Microfluidics (Oxford University Press, New York, 2005)

17. M.J. Madou, Fundamentals of Microfabrication (CRC Press, Boca Ratón, 2002)

18. F. Ojeda, R. Cuerno, R. Salvarezza, L. Vázquez, Phys. Rev. Lett. 84, 3125 (2000); F. Ojeda, R. Cuerno, R. Salvarezza, F. Agulló-Rueda, L. Vázquez, Phys. Rev. B 67, 245416 (2003)

19. P.M. Chaikin, T.C. Lubensky, Principles of Condensed Matter Physics (Cambridge University Press, Cambridge, 1995)

20. P.C. Hohenberg, B.I. Halperin, Rev. Mod. Phys. 49, 435 (1977)

21. G. Grinstein, Scale Invariance, Interfaces and Non-equilibrium Systems, edited by A. McKane, M. Droz, J. Vannimenus, D. Wolf (Plenum Press, New York, 1995)

22. R. Cuerno, M. Castro, Physica A 314, 192 (2002)

23. D.G. Cahill, J. Vac. Sci. Technol. A 21, S110 (2003)

24. See a recent overview in U. Valbusa, C. Boragno, F. Buatier de Mongeot, J. Phys. Condens. Matter 14, $8153(2002)$

25. S. Facsko, T. Dekorsy, C. Koerdt, C. Trappe, H. Kurz, A. Vogt, H.L. Hartnagel, Science 285, 1551 (1999); F. Frost, A. Schindler, F. Bigl, Phys. Rev. Lett. 85, 4116 (2000); R. Gago, L. Vázquez, R. Cuerno, M. Varela, C. Ballesteros, J.M. Albella, Appl. Phys. Lett. 78, 3316 (2001)

26. D. Walgraef, Spatio-Temporal Pattern Formation: With Examples from Physics, Chemistry, and Materials Science (Springer, New York, 1997)

27. F. Frost, B. Ziberi, T. Höche, B. Rauschenbach, Nucl. Instrum. Methods Phys. Res. B 216, 9 (2004)

28. M. Makeev, R. Cuerno, A.-L. Barabási, Nucl. Instrum. Methods Phys. Res. B 197, 185 (2002)

29. A. Bray, Adv. Phys. 43, 357 (1994)

30. W.H. Zurek, Phys. Rep. 276, 177 (1996); T.W.B. Kibble, in Patterns of Symmetry Breaking, edited by H. Arodz et al. (Kluwer Academic, New York, 2003)

31. P. Tejedor, P. Šmilauer, C. Roberts, B.A. Joyce, Phys. Rev. B 59, 2341 (1999)

32. P. Tejedor, M.L. Crespillo, B.A. Joyce, Appl. Phys. Lett. 88, 063101 (2006)

33. J.W. Evans, P.A. Thiel, M.C. Bartelt, Surf. Sci. Rep. 61, 1 (2006)

34. C. Schelling, G. Springholz, F. Schäffler, Thin Solid Films 369, 1 (2000) 
35. See contribution by M. Siegert to the volume in [21]

36. A. Karma, Phys. Rev. E 48, 3441 (1993); O. Pierre-Louis, C. Misbah, Phys. Rev. B 58, 2259 (1998); 58, 2276 (1998)

37. B. Davidovitch, E. Moro, H.A. Stone, Phys. Rev. Lett. 95, 244505 (2005)

38. H. Emmerich, The Diffuse Interface Approach in Materials Science (Springer, Berlin, 2003)

39. M.E.J. Newman, G.T. Barkema, Monte Carlo Methods in Statistical Physics (Oxford University Press, New York, 1999)

40. R. Cuerno, M. Castro, Phys. Rev. Lett. 87, 136103 (2001)

41. C.H.J. Van den Brekel, A.K. Jansen, J. Cryst. Growth 43, 364 (1977); B.J. Palmer, R.G. Gordon, Thin Solid Films 158, 313 (1988); G.S. Bales, A.C. Redfield, A. Zangwill, Phys. Rev. Lett. 62, 776 (1989)

42. H. Mori, Y. Kuramoto, Dissipative Structures and Chaos (Springer, Berlin, 1997)

43. M. Castro, R. Cuerno, J.G. Buijnsters, L. Vázquez, preprint (2006); R. Cuerno, M. Castro, in Proceedings of the 4th. European Congress on Computational Methods in Applied Sciences and Engineering, edited by P. Neittaanmäki et al. (ECCOMAS, Jyväskylä, 2004)

44. K. Sneppen, J. Krug, M.H. Jensen, C. Jayaprakash, T. Bohr, Phys. Rev. A 46, R7351 (1992)

45. M. Nicoli, M. Castro, R. Cuerno (2006) (preprint)

46. M. Castro, R. Cuerno, A. Sánchez, F. Domínguez-Adame, Phys. Rev. E 57, R2491 (1998);

M. Castro, R. Cuerno, A. Sánchez, F. Domínguez-Adame, ibid. 62, 161 (2000)

47. P. Schilardi, O. Azzaroni, R.C. Salvarezza, A.J. Arvia, Phys. Rev. B 59, 4638 (1999)

48. H.-C. Kan, S. Shah, T. Tadyyon-Eslami, R.J. Phaneuf, Phys. Rev. Lett. 92, 146101 (2004)

49. M.A. Auger, R. Gago, M. Fernández, O. Sánchez, J.M. Albella, Surf. Coat. Technol. 157, 26 (2002)

50. M.A. Auger, L. Vázquez, O. Sánchez, M. Jergel, R. Cuerno, M. Castro, J. Appl. Phys. 97, 123528 (2005); M.A. Auger, L. Vázquez, R. Cuerno, M. Castro, M. Jergel, O. Sánchez, Phys. Rev. B 73, $045436(2006)$

51. J.M. López, M.A. Rodríguez, R. Cuerno, Phys. Rev. E 56, 3993 (1997); J.J. Ramasco, J.M. López, M.A. Rodríguez, Phys. Rev. Lett. 84, 2199 (2000)

52. P. Sigmund, Phys. Rev. 184, 383 (1969); J. Mat. Sci. 8, 1545 (1973)

53. R.M. Bradley, J.M.E. Harper, J. Vac. Sci. Technol. A 6, 2390 (1988); R. Cuerno, A.-L. Barabási, Phys. Rev. Lett. 74, 4746 (1995)

54. M. Castro, R. Cuerno, Phys. Rev. Lett. 94, 139601 (2005)

55. O. Terzidis, P. Claudin, J.-P. Bouchaud, Eur. Phys. J. B 5, 245 (1998); A. Valance, F. Rioual, Eur. Phys. J. B 10, 543 (1999); Z. Csahók, C. Misbah, F. Rioual, A. Valance, Eur. Phys. J. E 3, 71 (2000)

56. R. Gago, L. Vázquez, O. Plantevin, T. H. Metzger, J. Muñoz-García, R. Cuerno, M. Castro, Appl. Phys. Lett. 89, 233101 (2006)

57. D. Flamm, F. Frost, D. Hirsch, Appl. Surf. Sci. 179, 95 (2001)

58. T. Frisch, A. Verga, Phys. Rev. Lett. 96, 166104 (2006) cond-mat/0607553 (preprint)

59. G.S. Bales, A. Zangwill, Phys. Rev. B 41, 5500 (1990)

60. M. Raible, S.J. Linz, P. Hänggi, Phys. Rev. E 62, 1691 (2000)

61. J. Muñoz-García, R. Cuerno, M. Castro, Phys. Rev. E 74, 050103(R) (2006)

62. M. Raible, S.G. Mayr, S.J. Linz, M. Mokse, P Hänggi, K. Samwer, Europhys. Lett. 50, 61 (2000); M. Raible, S.J. Linz, P. Hänggi, Phys. Rev. E 64, 031506 (2001)

63. J. Krug, Adv. Compl. Sys. 4, 353 (2001)

64. P. Politi, C. Misbah, Phys. Rev. Lett. 92 (2004) 090601; Phys. Rev. E 73, 036133 (2006)

65. A.A. Golovin, A.A. Nepomnyashchy, S.H. Davis, M.A. Zaks, Phys. Rev. Lett. 86, 1550 (2001)

66. O. Pierre-Louis, C. Misbah, Y. Saito, J. Krug, P. Politi, Phys. Rev. Lett. 80, 4221 (1998); J. Kallunki, J. Krug, Phys. Rev. E 62, 6229 (2000); F. Gillet, O. Pierre-Louis, C. Misbah, Eur. Phys. J. B 18, 519 (2000)

67. G. Danker, O. Pierre-Louis, K. Kassner, C. Misbah, Phys. Rev. E 68, 020601 (2003); Phys. Rev. Lett. 93, 185504 (2004)

68. M. Castro, J. Muñoz-García, R. Cuerno, M. García-Hernández, L. Vázquez, New J. Physics (2006) (submitted)

69. A.A. Nepomnhyaschy, Physica D 86, 90 (1995)

70. H.G.E. Hentschel, J. Phys. A: Math. Gen 27, 2269 (1994); J.M. López, M. Castro, R. Gallego, Phys. Rev. Lett. 94, 166103 (2005)

71. O. Pierre-Louis, Europhys. Lett. 72, 894 (2005) 